\title{
Estrategia de desarrollo endógeno: una propuesta para comunidades ecuatorianas
}

Endogenous development strategy: a proposal for Ecuadorian communities

Segundo Martin Quito Cortez. ${ }^{1}$, Dorian Damián Flores Aguilera. ${ }^{2} \&$ Yonimiler Castillo Ortega. ${ }^{3}$

\section{Abstract. \\ DOI: https://doi.org/10.33262/cienciadigital.v5i1.1518}

Introduction. The endogenous and exogenous development of a territory refers to the mechanism of its internal and external economic development, respectively. Several studies ensure that there is a high potential focused on the development of rural territories immersed in a scheme of transformation of their productive orientation, especially in places there are political guidelines that support employment and education, and emphasize endogenous development as a viable strategy for rural development. Objective. Develop an implementation strategy for a territorial development process based on endogenous principles that allows local governments and their communities to determine an action plan with defined lines of work in a participatory development process with a comprehensive approach. Methodology. The research is analytical, descriptive, and logical-deductive of a non-experimental type; the proposal is submitted to a validation process applying the Delphi method of expert judgment in the area of rural development and planning. Result. As a result, there is a strategy called DAPDEP (Diagnosis - Actors involved - community Policies - human and cultural DEvelopment Action plan, validated for its application in the Ecuadorian rural sector. Conclusion. The proposed DAPDEP strategy has an adequate assessment for its implementation, an

\footnotetext{
${ }^{1}$ Universidad Católica de Cuenca, Posgrado, Maestría en Desarrollo Local Mención en Ordenamiento Territorial, Cuenca, Ecuador, segundo.quito@ucacue.edu.ec, https://orcid.org/0000-0003-4717-4465

${ }^{2}$ Universidad Católica de Cuenca, Carrera de economía, Cuenca, Ecuador, dorian.flores@ucacue.edu.ec, https://orcid.org/0000-0003-0762-8928

${ }^{3}$ Universidad Católica de Cuenca, Posgrado, Maestría en Desarrollo Local Mención en Ordenamiento Territorial, Cuenca, Ecuador, ycastilloo@ucacue.edu.ec, https://orcid.org/0000-0002-7710-5199
} 
average of $86 \%$ experts validate the guide and $85 \%$ recommend its application in rural sectors of Ecuador, fulfilling the objective of the research and responding favorably to the research question posed.

Keyword: DAPDEP, rural development, endogenous development

\section{Resumen.}

Introducción. El desarrollo endógeno y exógeno de un territorio se refiere al mecanismo de su desarrollo económico interno y externo, respectivamente. Varios estudios aseguran que existe un potencial elevado enfocado el desarrollo de los territorios rurales inmersos en un esquema de transformación de su orientación productiva, especialmente en lugares existen lineamientos políticos que apoyen al empleo y a la educación, y enfatizan en el desarrollo endógeno como una estrategia viable para el desarrollo rural. Objetivo. Desarrollar una estrategia de implementación de un proceso de desarrollo territorial basado en los principios endógenos que permita a los gobiernos locales y sus comunidades determinar un plan de acción con líneas de trabajo definidas en un proceso de desarrollo de carácter participativo y con un enfoque integral. Metodología. La investigación es de tipo analítica, descriptiva, y lógico-deductiva de tipo no experimental; la propuesta es sometida a un proceso de validación aplicando el método Delphi de juicio de expertos en el área de desarrollo y planificación rural. Resultado. Como resultado se dispone de una estrategia denominada DAPDEP (Diagnostico - Actores involucrados Políticas comunitarias - Desarrollo humano y cultural - Plan de acción, validada para su aplicación en el sector rural ecuatoriano. Conclusión. La estrategia DAPDEP propuesta, tiene una valoración adecuada para su implementación, en un promedio de $86 \%$ los expertos validan la guía y en un $85 \%$ recomiendan su aplicación en los sectores rurales del Ecuador, cumpliendo el objetivo de la investigación y respondiendo favorablemente a la pregunta de investigación planteada.

Palabras clave: DAPDEP, desarrollo rural, desarrollo endógeno

\section{Introducción.}

El desarrollo endógeno busca el potencial de su territorio, ya sea económico, cultural o social; el desarrollo exógeno implica más inversión extranjera e innovación tecnológica. En las últimas dos décadas, el concepto de desarrollo económico ha sufrido cambios importantes. Frente al carácter de redistribución que presenta el desarrollo exógeno, que fomenta la captación de inversión extranjera para desarrollo económico de sectores periféricos. Siendo que el enfoque de desarrollo endógeno considera que la economía y el desarrollo las localidades y regiones puede darse empleando las potencialidades del mismo territorio, dicho enfoque ha ganado terreno y adeptos (Vázquez Barquero, 2000). La estrategia de desarrollo requiere un lineamiento político que debe motive el aprovechamiento de las circunstancias favorables del sector y dinamizar el uso adecuado 
de los recursos sean propios o externos. Esta no es una tarea fácil de lograr en una sociedad individualizada, heterogénea y exclusiva como la nuestra.

A pesar de esta premisa y del hecho de que algunos autores enfatizan en el desarrollo endógeno, se debe pensar en que la importancia de los recursos locales es relativa si hace falta contar con factores externos. Este hecho requiere que existan procesos emprendedores e innovadores en el que el territorio, a través de políticas adecuadas presente una estrategia propia con la que incidir en la dinámica económica de la localidad.

Las políticas públicas de desarrollo pueden ser determinadas de dos maneras: a) Desarrollo Endógeno, mediante el uso de recursos del sector, b) desarrollo exógeno mediante el uso de recursos provenientes de otros sectores productivos. Las políticas de desarrollo endógeno incentivan y promueven el uso y explotación de recursos propios, que se ejecuten bajo un control de normativas internas para beneficiar a las comunidades locales. Por otro lado, las políticas de desarrollo exógeno tienen como objetivo atraer, captar y emplear recursos externos para promover el desarrollo local. En estos casos, la iniciativa y el control de los recursos están mayormente fuera del alcance local.

Esto significa que deben realizarse dos tipos de acciones, por un lado y al inicio, sería necesaria la utilización de los recursos propios, así como de recursos externos en el desarrollo de la localidad, en segunda instancia, luego de alcanzado cierto nivel de desarrollo, se podrían impulsar de mejor manera y en mayor número a los proyectos de desarrollo endógeno que surjan en la localidad.

El modelo de desarrollo exógeno se basa principalmente en la utilización de recursos disponibles localmente que fueron obtenidos de otros sectores productivos o comunidades externas, y se basa en un aprovechamiento de experiencias de eses sectores. Esta estrategia exógena tiene algunas ventajas, que posibilitan su medición mediante indicadores específicos como son entre otros la inversión, el registro de ingresos y egresos, la tasa laboral, y la transferencia tecnológica. Sin embargo, una desventaja de este modelo de desarrollo es la dependencia del exterior y el capital en su mayoría se queda en el exterior (Guamán, 2014).

El desarrollo exógeno es posible mediante la implantación de factores externos en la comunidad o sector, mediante acción directa de empresas ya existentes en la localidad. El objetivo del desarrollo endógeno es crear empresas y está fuertemente relacionado con la actitud emprendedora de la sociedad.

Por otra parte, usar estrategias endógenas de desarrollo, actualmente es objeto de investigación y aplicación de políticas, de manera especial en los países latinos, ya que este nuevo modelo de desarrollo se caracteriza por la generar y fortalecer el capital social, 
difundir el conocimiento, promover el emprendimiento social, la descentralización y el empoderamiento de la comunidad.

Los procesos que se desarrollan bajo este modelo se desarrollan mediante el uso efectivo del potencial económico local y el compromiso de organismos locales y un adecuado fundamento legal enfocado al desarrollo. Desde este punto de vista endógeno, la sociedad y la economía están integradas (Arocena, 1995), citado por (Cordero, 2018). "Desde la perspectiva del desarrollo endógeno, cada espacio económico aparece con una configuración propia, que se ha ido definiendo en función de los sucesivos sistemas productivos, de los cambios tecnológicos y organizativos de las empresas e instituciones y de las transformaciones en el sistema de relaciones sociales e industriales." (Vázquez Barquero, 2000).

El desarrollo endógeno debe ser entendido como el desarrollo local que se genera principalmente por iniciativas locales, se basa principalmente en los recursos propios de cada región (Picci, 1993). Este modelo de desarrollo se enfoca a la potenciación de los recursos y valores propios de una comunidad. Investigaciones referentes al desarrollo endógeno sugieren seguir una estrategia bien definida que integre adecuadamente los recursos y valores culturales propios legalmente disponibles y explotables para lograr el crecimiento económico esperado (Hoogvelt, 1978).

Este artículo inicia con una contextualización donde se analiza el entorno ambiental, tendencias de desarrollo, estrategias y casos de éxito endógenos para desarrollo rural, se establece la metodología de investigación, seguido de la estrategia propuesta denominada DAPDEP, con la validación y las conclusiones obtenidas, así como una definición de posibles trabajos futuros.

\section{Contextualización.}

Al 2018, en el Ecuador, la pobreza alcanza el 43\%, la pobreza por nivel de ingresos alcanzó un $24.5 \%$, y la extrema pobreza extrema alcanzó el 9\%, por ello, el gobierno ecuatoriano se encuentra desarrollando estrategias que permitan mejorar esos porcentajes de pobreza, que influye directamente al desarrollo rural. Aunque América Latina tiene considerables avances económicos y sociales, estas mejoras no tienen un comportamiento de equidad territorial, existen aún, diferencias muy marcadas en cuanto al desarrollo social e inclusive al desarrollo humano en varios países, mostrando su mayor afección en territorios con menor población del sector rural, y en mayor escala en poblaciones originarios o afrodescendientes. (CLDR, 2019) 
Si cada sociedad tiene la capacidad de promover un proceso de mejoramiento interno, se convierte en una situación de desarrollo endógeno, que comienza cuando cada territorio tiene a disposición recursos económicos, humanos, culturales e institucionales de escala sin explotar. El desarrollo endógeno otorga a las organizaciones, instituciones y empresas locales, así como a la sociedad civil una función primordial en el proceso de crecimiento y de cambios estructurales (Vázquez Barquero, 2000), este hecho permite la generación y establecimiento de nuevas organizaciones productivas y por tanto la generación de nuevas plazas de empleo. En este proceso, la sociedad se integra en la economía.

El desarrollo endógeno debe concebirse como un proceso continuo de cambio estructural y desarrollo comunitario, en el cual los lineamientos y normativas aplicado en actividades productivas, la colaboración social, el proceso de formación y gestión cultural, son los factores que orientan el proceso de cambio en la localidad.

El Código Orgánico de Organización Territorial Autonomía y Descentralización, el desarrollo enfocado en factores endógenos, se basa en una nueva forma organizativa estatal, que conduce a los gobiernos regionales a participar más en la asignación del presupuesto nacional y a da a los gobiernos locales una mayor autonomía que va de la mano con una mayor capacidad financiera (COOTAD, 2010).

La investigación conducida por Woods, señala que en a finales del siglo pasado, se originaron cambios importantes en el desarrollo rural europeo. De hecho, dichos cambios hicieron que se dejen de lado los métodos exógenos tradicionales y los métodos sectoriales basados en criterios de modernización, y que se adopten métodos tradicionales y locales, fortaleciendo el enfoque endógeno e integral, fundamentando el desarrollo local en objetivos ambientales, sociales y económicos (Woods \& McDonagh, 2011).

\section{Tendencias de desarrollo rural.}

La teoría de este método de desarrollo rural, especialmente en las últimas dos décadas, ha logrado importantes avances y ha recibido contribuciones de diferentes disciplinas y perspectivas. Resultados obtenidos a partir de investigaciones anteriores (Guinjoan, 2017), han establecido un trabajo orientado a desarrollo rural para que éste se adapte a la situación y al contexto actual. En ese sentido, la investigación propuesta en este estudio plantea un análisis de estrategias y objetivos de desarrollo rural, con la finalidad de aclarar el debate eterno sobre el significado real del desarrollo rural.

Aunque las personas generalmente ven el mundo rural como una realidad estable, el hecho es que las áreas rurales, se quiera o no, tienen un comportamiento dinámico, afectado frecuentemente por influencias externas que pretenden transformar sus bases 
culturales y su comunidad social (Hoggart \& Paniagua, 2001). El final de la Segunda Guerra Mundial marca una transformación de los sectores rurales, otorgándoles importancia y mayor atención, pues estos sectores empezaron a verse afectados por la consolidación de los modelos urbanos e industriales, que enfocaron todos sus esfuerzo y generaron una tendencia industrial en un sistema en serie de producción; con ello la población, los recursos y la inversión sufrieron una redistribución, dando como resultado una reestructuración territorial en centros y las áreas circundantes de las ciudades a escala regional y global (Oliva, 1997).

Según (Quispe Fernández, 2016), además de los modelos de desarrollo local existentes, el modelo enfocado en factores endógenos es considerada una estrategia alternativa que puede ser puesto en práctica por medio de políticas adecuadas, principalmente para los países del sur de nuestro continente.

El desarrollo endógeno se originó en Europa, como un paradigma dentro de los esfuerzos de desarrollo económico que se ha formado en las últimas décadas, especialmente desde la década de 1980 (Vázquez, 2007). A partir de ese paradigma, durante el período mencionado se han desarrollado dos orientaciones investigativas: la primera que surgió como un intento por encontrar una definición de desarrollo que permita el accionar público en favor del desarrollo de regiones atrasadas (Quispe Fernández, 2016); la segunda línea de investigación es empírica, y se desarrolló como resultado de la búsqueda de una explicación a los procesos del desarrollo industrial en el sur de Europa (Vázquez Barquero, 1983), citado en (Vázquez Barquero, 2018); esta línea de investigación revela las experiencias causadas por la modernización impulsada por factores internos, enfatizando la solución de problemas de la denominada "sociedad industrial" en lugar de copiar los procesos de industrialización de acuerdo con pautas preestablecidas. Por lo tanto, en base a la experiencia interna europea y a la investigación realizada bajo estas líneas de investigación, se extrajeron conceptos útiles para el análisis y la acción de la comunidad local.

Es importante comprender el modelo de desarrollo endógeno, a fin de comprender cómo usarlo para analizar el proceso de desarrollo, independientemente la tasa de desarrollo, y que puede ser aplicado en cualquier tipo de economía de países en desarrollo o de países avanzada dos. Es importante entonces, la conformación de teoría y práctica en estrategias endógenas, por un lado la teoría indica el proceso y el mecanismo de gestión de los mismos en comunidades, y la práctica indica las experiencias de la aplicación de políticas enfocadas al desarrollo (Quispe Fernández, 2016).

Desde la década de 1960, se han cuestionado los estándares económicos y su preponderancia en el desarrollo social, cuestionamientos que condujeron a la aparición de tendencias que criticaron tanto al paradigma de modernización como a sus políticas de 
desarrollo exógeno, aduciendo su orientación mayormente económica y no social (Pike et al., 2007). En este sentido, se debe citar a actores como el economista Celso Furtado, quien propuso en 1961 reinterpretar el desarrollo de tal manera que éste incluya modificaciones en su estructura y considere valores sociales, para de esta manera apostar por un modelo endógeno (Furtado, 1961).

En este orden de ideas, alrededor de 1965, promovidas por los gobiernos locales y con el impulso de algunas áreas rurales francesas surgieron las primeras estrategias de desarrollo, con el objetivo de reducir la caída de las actividades en un país altamente intervencionista y centralizado (Dalla, 1999). Unos años más tarde, la crisis económica presentada en la década de 1970 mostró que la centralización de las políticas regionales de carácter modernizador, no podían promover el desarrollo en las áreas circundantes, porque la economía de las regiones periféricas seguía dependiendo de la toma de decisiones externas (Capellin, 1992). Por lo tanto, en un contexto de reajuste estructural económico global y con notables diferencias regionales, las comunidades locales comenzaron a organizarse para enfrentar nuevos desafíos internacionales a partir de sus propias iniciativas (Stohr, 1992), citado en (Antti et al., 2008).

\section{Estrategias de desarrollo rural endógeno.}

Luego del análisis sobre en qué consiste el mecanismo y el desarrollo del modelo de desarrollo endógeno, se debe destacar que, siendo un término aun confuso, se destaca que existen importantes particularidades entre los mecanismos y estrategias de desarrollo que han surgido en base a este modelo y que estas diferencias dependen de varios puntos de vista o interpretaciones desde los que se aborda el desarrollo endógeno. En este sentido, según se indica en (Quispe Fernández, 2016), se pueden distinguir los siguientes puntos de vista desde los cuales considerar al desarrollo endógeno:

$\checkmark$ Punto de vista evolutivo. - Según este criterio, la estrategia de desarrollo basado en factores endógenos se entiende como un proceso de generación de capital y de tecnología, que justifica por qué ocurre el proceso de desarrollo. Está relacionado con el mecanismo que permite un desarrollo local y enfocado en teorías económicas sostenibles, es decir, el desarrollo social y el desarrollo económico está intrínsecamente relacionado con el desarrollo endógeno y el desarrollo económico. Además, desde este enfoque se argumenta que los recursos utilizados en proyectos de inversión afectan el poder del desarrollo, por lo tanto, contribuyen a la aparición de mayores rendimientos, entonces son posibles los cambios (evoluciones) económicos, sociales e institucionales.

$\checkmark$ Punto de vista estratégico. - El desarrollo endógeno, se entiende como un modelo alternativo que se incorpora en la localidad mediante políticas adecuadas de 
desarrollo, valorando iniciativas propias basadas en ejes culturales o religiosos que han surgido en las últimas décadas de forma espontánea en sectores y comunidades a nivel mundial, dichas iniciativas son una reacción por parte de los actores de la localidad frente a los desafíos que trae consigo la globalización y sus problemas anexos especialmente el desempleo y el incremento de niveles de pobreza crónica en nuestro continente. Desde este punto de vista, el desarrollo endógeno posee características políticas y sociales porque puede usarse fácilmente de forma politizada.

$\checkmark$ Punto de vista cultural. - El desarrollo endógeno, es esta orientación, el ser humano es elemento central, y junto a ello su creatividad y sus capacidades sirven de base para el progreso de las localidades, aunando el hecho de que es el propio beneficiario del cambio económico y social resultante, hecho que hace al desarrollo endógeno un tema culturalmente sostenible. Teniendo esto en cuenta, el desarrollo es el resultado de la utilización de la creatividad empresarial de los ciudadanos frente a los cambios ambientales en cualquier momento. Desde esta perspectiva, el desarrollo endógeno es de naturaleza benéfica: al promover el desarrollo económico y social sostenible.

$\checkmark$ Punto de vista territorial. - El desarrollo endógeno se concibe desde esta visión como un proceso que transforma y diferencia a la producción, esta concepción se basa en "la idea de que cada comunidad territorial se ha ido formando, históricamente, en función de las relaciones y vínculos de intereses de sus grupos sociales, de la construcción de una identidad y de una cultura propia que la diferencia de las otras comunidades" (Massey, 1995), citado también en (Docampo, 2007). Con esto en mente, una comunidad territorial puede generar de manera proactiva nuevos proyectos e iniciativas que empleen sus propios recursos y exploten las capacidades de los habitantes locales como una solución efectiva a sus problemas y aportar a suplir sus requerimientos y necesidades. Justamente, son los actores de la localidad, a través de sus proyectos, iniciativas e innovaciones, conjugado con un trabajo colaborativo y participativo enfocado en la generación y gestión de nuevos lineamientos políticos contribuirán de esa manera para lograr la dinamización e impulsar el crecimiento productivo de local y comunitario del país. Considerando así que el desarrollo endógeno tiene características sociales, económicas y políticas.

$\checkmark$ Punto de vista del desarrollo autónomo. - En este punto de vista, se concibe a las estrategias endógenas de desarrollo como el proceso de generación de capital, y su equidad, justicia e igualdad, "asociado con la capacidad de una comunidad local para utilizar el potencial de desarrollo existente en el territorio y dar respuesta a los desafíos que se le plantean en un momento histórico determinado" (Vázquez, 2007). El desarrollo endógeno es importante para la autogestión dentro de la comunidad. 
Punto de vista productivo. - El desarrollo endógeno se concibe como el proceso donde la comunidad emplea y aprovecha las capacidades del territorio acorde con los sistemas de producción vigentes, a partir de ello se puede alcanzar en gran medida el nivel de desarrollo deseable (ideal) según el buen vivir.

$\checkmark$ Punto de vista populista. - Bajo esta visión, es un proceso que acumula capital que se centra en el estado, por este motivo este modelo de desarrollo contempla el uso de sus propios recursos, por lo que puede aplicarse en cualquier lugar, territorio o región, hecho que se hace posible ya que todos los territorios tienen potencial de desarrollo. El problema radica en el diseño de proyectos que sean propuestos y gestionados por las organizaciones y demás actores de la comunidad.

$\checkmark$ Punto de vista humano. - El modelo de desarrollo endógeno debe concebirse como un proceso de desarrollo humano, es un proceso donde los comuneros y actores productivos del sector priorizan sus ideales y proyectos, y planean su desarrollo. De esta forma, la capacidad productiva de las localidades, territorios y regiones que estimula las fuerzas de desarrollo es capaz de responder a este ideal de desarrollo.

Finalmente, se determina la necesidad del aporte fundamental del gobierno local como entidad de atención directa a las necesidades no satisfechas de la comunidad. Este aporte significa: a) Brindar acompañamiento a los procesos innovadores de desarrollo, b) Fortalecer a los a los actores productivos un visión empresarial, c) Generar entornos y mecanismos adecuados de comunicación con instituciones públicas y privadas que permitan la creación de redes colaborativas de desarrollo conjunto, d) Promover conceptos basados en cooperativismo y redes asociativas para beneficios comunes, e) Promover redes colaborativas de desarrollo (Segura \& Sanchez, 2003)

Las tendencias actuales de la dinámica territorial, se enfoca en una tendencia a descentralizar y fragmentar los sectores rurales con una invasión industrial o no, fortaleciendo algunas actividades productivas y dispersando otras según se oriente la actividad empresarial como tal; existe un tendencia de dispersión social por la generación de diversas actividades terciarias, y por una acumulación de actores en los ejes de relación, ocasionando crecimientos inequitativos de los sectores, finalmente una tendencia creciente en afecciones al medio ambiente.

\section{Casos de desarrollo rural endógeno.}

En el contexto sudamericano es menester mencionar algunas investigaciones que se ocupan del tema siendo las más relevantes las siguientes: 
Desarrollo endógeno: Agroecología y agricultura campesina. - En este trabajo, los investigadores presentan una aproximación teórica sobre el desarrollo endógeno y su relación a la agricultura y la agroecología de los campesinos de la Rinconera, en el estado de Apure, Venezuela. Se indica que el estudio fue aplicado principalmente a campesinos líderes familiares y que las entrevistas ejecutadas permitieron evaluar indicadores que se consideran importantes en la generación del fundamento teórico sobre los entornos de desarrollo endógeno, por medio de los principios agroecológicos, siendo importante mencionar que los informantes del estudio están convencidos de que el desarrollo de la comunidad está fuertemente relacionado con los procesos de formación de la comunidad, hecho debe ir acompañado políticas inclusivas que a parte de la formación requerida permitan el acceso a los recursos locales y a las herramientas tecnológicas adecuadas para suplir necesidades insatisfechas de las familias de esta localidad, considerando el cuidado del medioambiente (Solano, 2017).

$\checkmark$ Modelo de desarrollo para comunidades de costa rica. - Como solución alternativa a los problemas de carácter social, aspectos de influencia económica y ambiental específicamente en el sector rural de Costa Rica, los autores de este trabajo proponen un modelo sostenible de desarrollo rural basado en factores endógenos (DRES), que se fundamenta en el uso de ecotecnologías para una gestión adecuada de residuos que generen actividades productivas. La propuesta se percibe como un emprendimiento innovador, que permite a las comunidades locales mejorar sus condiciones de vida mediante la utilización de sus propios recursos, sin cerrar la posibilidad de integrar y/o modificar conocimientos externos a la comunidad. Este modelo se centra en el empleo de las habilidades que han adquirido y desarrollado los habitantes de la comunidad, o a su vez por la obtención de nuevas competencias por parte de estos para mejorar las condiciones de vida (Chassoul-Acosta \& Charpentier, 2018).

$\checkmark$ Metodología de gestión estratégica en el planeamiento de desarrollo rural, el caso comunidad mi Perú, 2017-2021. - En esta investigación, se establece una metodología de gestión para el planeamiento de desarrollo rural para comunidades del Perú, compuesta de técnicas y métodos en función de los tres pilares de desarrollo que se adapte a las particularidades de cada comunidad y su contexto. Este trabajo plantea además, una alternativa viable de un plan para el desarrollo de comunidades rurales denominado Comunidad Mi Perú, para el período 2017 - 2021 (Mendoza, 2018).

$\checkmark$ Experiencias de desarrollo rural basado en factores endógenos en Ecuador. - En el caso de nuestro país, varios esfuerzos han generado proyectos y experiencias de desarrollo endógeno, algunos de ellos se mencionan en (Torres Dávila et al., 2011), en este trabajo, se presenta una síntesis de algunos procesos económicos, de salud, 
educativos, de derechos sociales, etc. Realizados por organizaciones de base, agencias de cooperación internacional e instituciones de desarrollo en el territorio ecuatoriano. Luego de presentar un estudio introductorio, esta compilación presenta 13 artículos, cada uno se corresponde a un trabajo de tesis de profesionales maestrantes (2007-2009), en la Universidad Politécnica Salesiana. Estos trabajos presentan una visión de dichas experiencias, mismas que se consideran importantes ya que la mayoría de los tesistas de esa cohorte, son funcionarios de la Organización internacional Ayuda en Acción (AeA), la cual impulsa varias experiencias de desarrollo endógeno en el Ecuador por lo que, estos trabajos son en cierto grado una retrospectiva del trabajo realizado en este ámbito en nuestro país, entre los trabajos más importantes existen enfocados en bienestar con procesos activos de ayudas directas(Torres, 2010); estudios enfocados a políticas crediticias y su impacto en sectores específicos del norte del país (Herrán, 2011), y en otros sectores de la costa oeste del país (Narea, 2011); otros estudios enfocados en la parte educativa de formación social (Montesdeoca, 2011); investigaciones profundas en aspectos productivos y comerciales(Cazares, 2010); investigaciones en términos de recate y reinserción social (Galárraga, 2011), en apoyo a niños de la calle (Jaramillo, 2011), y un apoyo directo al desarrollo de capacidades endógenas (Santana, 2011); investigaciones en el fomento de redes productivas agropecuarias(Benítez, 2011), y fomento a actividades turísticas(Zambrano, 2011); y finalmente estudios enfocados a fortalecimiento de capacidades de adultos(De la Fuente, 2011), fortalecimiento de servicios de salud (Guailla, 2011), y cuidado infantil (Navarrete, 2011), inclusive capacitación en procesos industriales, El (Benavides, 2011).

\section{Metodología.}

Partiendo de una de las concepciones de ciencia definida como un conjunto de información recolectada de una mantera metódica, sistemática y comprobable, aplicando técnicas como la observación y con un proceso de análisis sistemático se deducen principios y leyes generales (ASALE \& RAE, 2020) la propuesta de investigación se apoya en el método científico y sus pasos tal como se describe a continuación:

- Revisión Bibliográfica. - La revisión de literatura especializada que permite determinar el estado actual del problema y posibles soluciones.

- Pregunta de Investigación. - ¿Una estrategia de implementación de un proceso de desarrollo territorial basado en los principios endógenos permitirá a los gobiernos y comunidades locales, apoyar a definir mecanismos participativos de desarrollo integral?

- Propuesta. - Esta fase, permite al investigador, basado en el fundamento teórico y en un análisis de casos de éxito, se construye una estrategia de implementación de un proceso de desarrollo territorial basado en los principios endógenos aplicable al entorno ecuatoriano. La propuesta será sometida a un proceso de 
validación formal, utilizando el Método Delphi de Juicio de expertos, que facilita la obtención de opiniones de expertos que están relacionados directamente con el objetivo de esta investigación ; este método es aplicado cuando la evidencia empírica de la investigación es escasa, y adicionalmente, es un método que recoge información confidencial a los expertos, lo que permite expresarse con independencia; disminuye inconvenientes de representatividad y dominio sobre el tema.

- Validación. - En esta etapa se procesa la información recabada para la validación de la estrategia propuesta y se analiza los datos con estadísticamente.

- Comunicación. - En esta etapa, se pondrán a consideración de una revista especializada los resultados de la investigación.

\section{Propuesta: DAPDEP - Una estrategia viable}

Con el fundamento teórico analizado, se propone una estrategia de implementación de un proceso de desarrollo territorial basado en los principios endógenos denominada

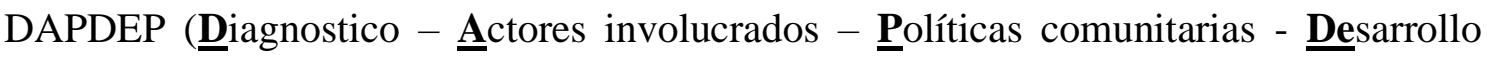
humano y cultural - Plan de acción) para el territorio ecuatoriano.

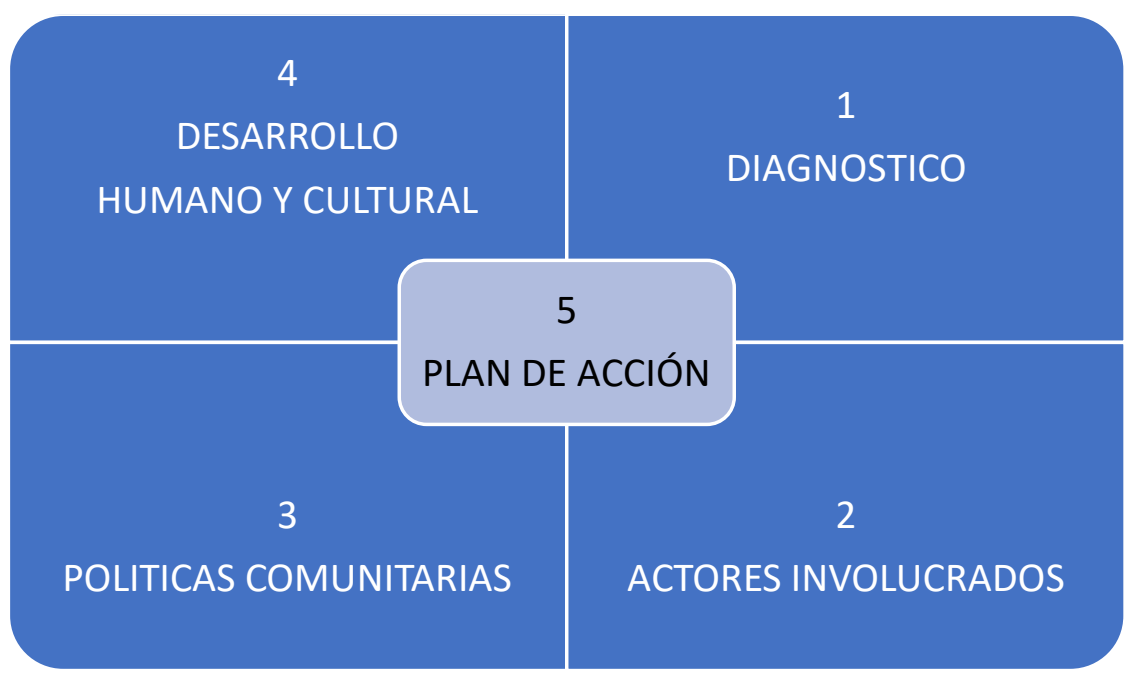

Figura 1: Esquema general estrategia DAPDEP Elaborado por el autor

\section{1.- Diagnostico}

En un propósito de elevar la calidad de vida de la población, es necesario replantear la actual organización del territorio y establecer una distribución regional adecuada de las actividades económicas, propiciando una igualdad de oportunidades a todos los habitantes del territorio. 
Para cumplir esta fase de la estrategia se debe considerar los siguientes estudios:

- Determinación de las características del medio ambiente, en aspectos como geomorfología, edafología e hidrología, con el objeto de conocer las capacidades y aptitudes del entorno.

- Determinar el suelo y sus usos, y el manejo de aspectos como red vial, transporte, logística, limites naturales y fronteras culturales; determinar un inventario de atractivos naturales y su accesibilidad, un análisis de factores de riesgo y efectos contaminantes.

- Determinar la apertura comunitaria para receptar programas de desarrollo y tendencias organizativas del territorio.

- Determinar el entorno social comunitario, en un análisis de indicadores de población, nivel educacional, servicios de salud, condiciones de infraestructura de viviendas, ratios demográficas, tasa de desempleo, subempleo y empleabilidad, índices de pobreza, niveles de seguridad social, y estructura de sociedad civil.

- Determinar restricciones y limitaciones legales

\section{2.- Involucrados}

El recurso humano endógeno y exógeno son los principales actores que intervienes en la implementación de estrategias desarrollo, se debe considerar que la responsabilidad y participación es de las instancias gubernamentales, y de todos los actores que se desenvuelven en el territorio, con una activa participación en las estrategias de solución a las necesidades insatisfechas de la comunidad. Entonces, justamente son los actores y líderes políticos, autoridades económicas, líderes sociales y culturales quienes disponen de proyectos y alternativas que tienden a un aprovechamiento adecuado de los recursos y permitan potencializar la comunidad, propiciando establecer parámetros mínimos de calidad, minimizando la afección ambiental, cultural y social. (Arocena; 1995).

Son los actores cuya principal función es promover proyectos y programas de desarrollo. (Alburquerque, 2003).

Entre los actores a considerar está:

- La comunidad. - Líderes comunitarios, empresas locales, gremios, ONGs, Instituciones educativas

- Instituciones de gobierno. - El gobierno nacional, los Gads Parroquiales, municipales, y provinciales,

- Equipo técnico exclusivo para capacitación, asesoría y seguimiento en el proceso. 


\section{3.- Políticas de desarrollo endógeno}

Parar neutralizar las consecuencias de una globalización directamente influyente en el desarrollo comunitario, especialmente en comunidades con economías emergentes o de débil desarrollo, se plantean políticas de desarrollo y aplicación de estrategias basados en principios endógeno (Alburquerque, 2003).

Entre las políticas mundialmente aplicadas se encuentran

- Promover políticas de redes empresariales (Vázquez Barquero, 2005).

- Establecer procesos formativos de innovación y capacidades específicas para un fortalecimiento del proceso productivo

- Dotación de entornos favorables para el desarrollo en términos de equipamientos e infraestructuras.

- Implementación de mecanismos enfocadas a elevar la eficiencia organizativa y de gestión

- Comercialización enfocada en los aspectos culturales y de patrimonio

- Perfeccionamiento y validación de técnicas artesanales

- Fortalecimiento de explotación de productos propios.

\section{4.- Desarrollo humano y cultural}

El desarrollo endógeno, tiene su entorno favorable en sectores y comunidades donde las instituciones estimulan el crecimiento económico, y sus procesos sociales y culturales apoyan el progreso de su comunidad (Weber, 1905; Fukuyama, 1995), (Landes, 1998; North, 1990). Fundamentalmente los procesos de desarrollo se ven influenciados por factores culturales, así como por las normativas que regulan los procesos de acumulación de capital y las interacciones entre organizaciones y personas del sector. Entre las estrategias para el desarrollo humano y cultural se determinan:

- Formación y concientización de los ciudadanos, en aspectos culturales, conductuales, creativos y emprendedores.

- Desarrollo, creatividad y capacidad empresarial con proceso continuo de dinamismo económico.

- Desarrollo, culturalmente sostenible con especial atención en definiciones y capacitación en estrategas económicas, motivación en procesos innovadores, dinamismo tecnológico, y cambio estructural de organizaciones.

\section{5.- Plan de acción}


Con la información y las consideraciones mencionadas, estableciendo un entorno adecuado tanto de la comunidad como de los actores gubernamentales, se puede establecer un flujo de acciones a cumplir:

- Programas de capacitación. - Para lograr una transferencia de tecnología aplicable a la realidad del territorio

- Organización de productos y servicios. - Según estudio de mercado

- Organización de procesos productivos. - Según factibilidad del territorio

- Planes de difusión nacional e internacional. - Promoción y fortalecimiento de los productos y servicios.

- Promoción turística nacional e internacional. - Potenciando las características culturales y aspecto de valor territorial

- Evaluación continua de impactos. - Evaluación de impactos en desarrollo económico, social, y cultural.

- Plan de mejoras. - Para un mejor aprovechamiento de las potencialidades del territorio.

\section{Validación.}

Para la validación de la estrategia de implementación de un proceso de desarrollo territorial basado en los principios endógenos, se realiza una encuesta ${ }^{4}$ y se aplica el método de juicio de expertos.

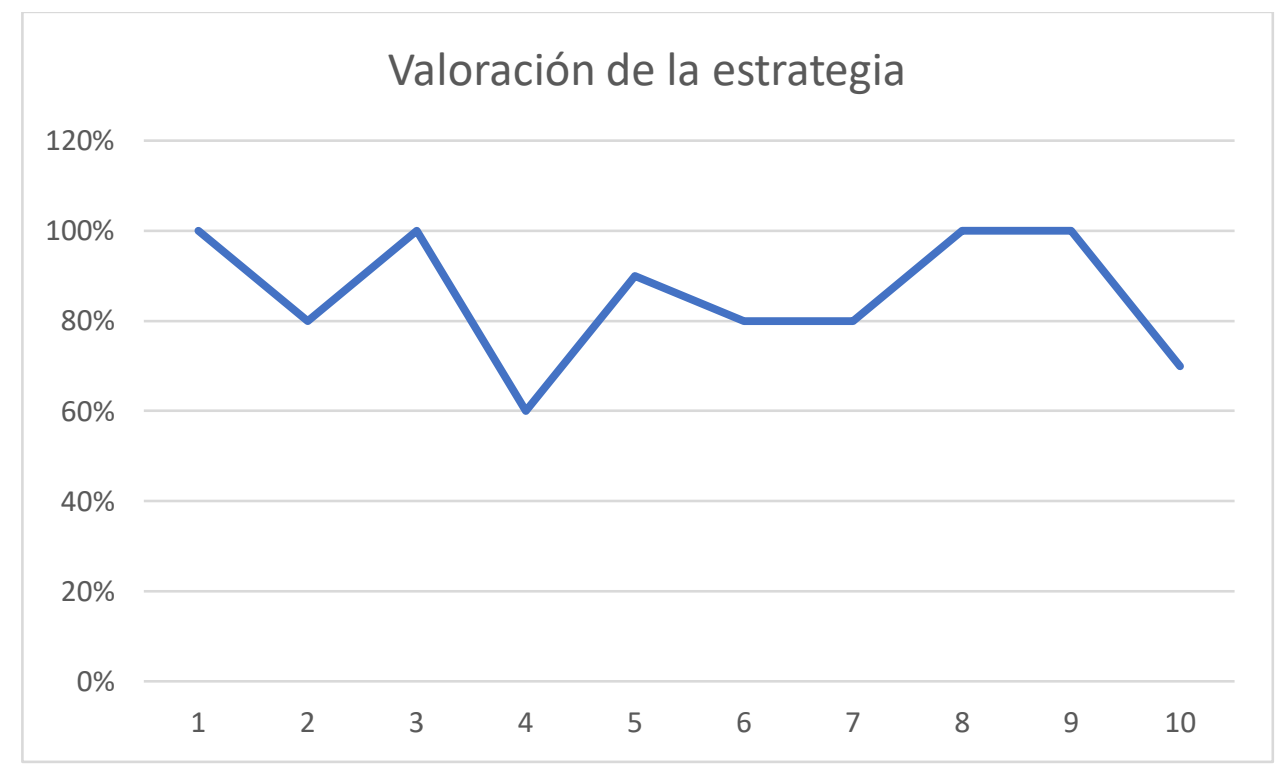

Gráfico 1: Esquema general estrategia DAPDEP Elaborado por el autor

\footnotetext{
${ }^{4}$ https://forms.gle/5NVTdkeisztmeNCYA
} 
De ello se obtiene que el 100\% de encuestados considera necesario fortalecer el desarrollo rural de comunidades ecuatorianas, el $80 \%$ considera que es adecuada la implementar de estrategias endógenas para desarrollo rural y el $20 \%$ considera aplicar otras estrategias, el $100 \%$ Considera altamente importante realizar un diagnóstico de territorio antes de implementar un proceso de desarrollo territorial basado en los principios endógenos, el $60 \%$ considera altamente importante la definición de actores e involucrados en un proceso de desarrollo territorial basado en los principios endógenos, sin embargo el $90 \%$ considera altamente importante el establecimiento de compromiso de dichos actores, el 80\% Considera altamente importante la definición de políticas comunitarias y el establecimiento de planes y programas de desarrollo humano y cultural, mientras que el 100\% Considera altamente importante la definición de plan de acción integral para implementar la estrategia.

En cuanto a la estrategia y su aplicabilidad, el 100\% de encuestados recomendaría aplicar la estrategia propuesta denominada DAPDEP en los sectores rurales del Ecuador y un 70 $\%$ Considera recomendable la aplicación de la estrategia propuesta DAPDEP, y recomiendan incluir otros aspectos necesarios en un proceso de desarrollo territorial basado en los principios endógenos, los cuales serán considerados por su complejidad de implementación en un trabajo futuro.

\section{Conclusiones.}

- Es fundamental disponer de una estrategia de desarrollo basado en principios endógenos que permita a los gobiernos locales y sus comunidades, definir sus estrategias en miras a un desarrollo local integral de manera participativa.

- La revisión bibliográfica y el análisis de caso de éxito en temas de desarrollo rural, permitió crear una estrategia de implementación de un proceso de desarrollo loca basado en los principios endógenos.

- La estrategia DAPDEP propuesta, tiene una valoración adecuada para su implementación, en un promedio de $86 \%$ los expertos validan la guía y en un $85 \%$ recomiendan su aplicación en los sectores rurales del Ecuador.

\section{Trabajos futuros.}

- Análisis de impacto de del desarrollo rural con estrategias endógenas en la migración de habitantes del territorio.

- Estudio sobre estrategias específicas de segundo momento.

- Modelo presupuestario para implementación de estrategias endógenas en desarrollo rural 
- Estudio de mecanismo de intervención en la dinámica cultural y económica en sectores rurales.

\section{Referencias Bibliográficas.}

Antti, S., Katja, R., \& Timo, L. (2008). Local Governance and Flexible Lifestyle in Rural Regions.

Alburquerque, F. (2003). Teoría y práctica del enfoque del desarrollo local. Instituto de Economía y Geografía. Consejo Superior de Investigaciones Científicas, Madrid, $1-24$.

ASALE, R.-, \& RAE. (2020, junio 19). Ciencia | Diccionario de la lengua española. «Diccionario de la lengua española» - Edición del Tricentenario. https://dle.rae.es/ciencia

Arocena, J. (1995). El desarrollo local: Un desafío contemporáneo. Nueva Sociedad. http://www.ecominga.uqam.ca/ECOMINGA_2011/PDF/BIBLIOGRAPHIE/GU IDE_LECTURE_1/2/2.Arocena.pdf

Benavides, L. (2011). El procesamiento de la 'papa chips' en la escuela La Unión de Saguazo. Universidad Politécnica Salesiana. https://dspace.ups.edu.ec/

Benítez, V. (2011). Los productores agropecuarios en el Ad Morona organizados bajo una estrategia de red. Universidad Politécnica Salesiana. https://dspace.ups.edu.ec/

Capellin, R. (1992). Theories of Local Endogenous Development and International Cooperation. Development Issues and Strategies in the New Europe: Local, Regional and Interregional Perspectives, 1-19.

Cazares, L. (2010). Producción y comercialización asociativa de leche en la zona de IntacImbabura. Universidad Politécnica Salesiana. https://dspace.ups.edu.ec/

Chassoul-Acosta, M. J., \& Charpentier, C. (2018). Modelo de desarrollo sostenible para tres pequeñas comunidades en Costa Rica. Revista de Ciencias Ambientales, 52(2), Article 2. https://doi.org/10.15359/rca.52-2.10

COOTAD. (2010). CODIGO ORGANICO ORGANIZACION TERRITORIAL AUTONOMIA DESCENTRALIZACION. Registro Oficial Suplemento 303, 201.

Cordero, J. (2018). El gobierno municipal. ¿Promotor del desarrollo local? El caso del municipio semiurbano de Cd. Ixtepec, Oaxaca. Estudios sociales. Revista de 
alimentación contemporánea y desarrollo regional, 28(52), 0-0. https://doi.org/10.24836/es.v28i52.575

Dalla, G. (1999). Algunas reflexiones sobre la metodología del Desarrollo Local a partir del ejemplo francés. Manual de Desarrollo Local, 1999, ISBN 84-95178-22-2, págs. 33-41, 33-41. https://dialnet.unirioja.es/servlet/articulo?codigo=2907063

De la Fuente, M. (2011). El programa de alfabetización "Yo sí Puedo". Universidad Politécnica Salesiana. https://dspace.ups.edu.ec/

Docampo, M. G. (2007). Perspectivas Teóricas en Desarrollo Local. Netbiblo.

Furtado, C. (1961). Desenvolvimento e subdesenvolvimento. Editora Fundo de Cultura. https://repositorio.cepal.org/bitstream/handle/11362/1616/S33098N962Av1.pdf? sequence $=1$

Fukuyama, F. (1995). Trust: The Social Virtues and the Creation of Prosperity. Nueva York: The Free Press.

Galárraga, W. (2011). La reinserción social y familiar de los chicos de la calle atendidos en el proyecto salesiano de Quito. Universidad Politécnica Salesiana. https://dspace.ups.edu.ec/

Guailla, I. (2011). La red de servicios de salud en el cantón Echeandía. Universidad Politécnica Salesiana. https://dspace.ups.edu.ec/

Guamán, M. (2014). Cantón Paltas: Estrategias para su desarrollo económico y social. Pontificia Universidad Católica del Ecuador. http://repositorio.puce.edu.ec:80/xmlui/handle/22000/6857

Guinjoan, E. (2017). Perseguint les claus de l'èxit: Una anàlisi comparativa de tres experiències de desenvolupament rural: el Priorat, el Lluçanès i la Conca de Tremp. https://ddd.uab.cat/record/176187

Herrán, J. (2011). El microcrédito como gestión compartida: La experiencia de la Casa Campesina Cayambe. Universidad Politécnica Salesiana. https://dspace.ups.edu.ec/

Hoggart, K., \& Paniagua, A. (2001). What rural restructuring? Journal of Rural Studies, 17(1), 41-62.

Hoogvelt, A. M. M. (1978). The Sociology of Developing Societies. Macmillan International Higher Education.

Jaramillo, E. (2011). La escuela de ciudadanía del proyecto salesiano "Chicos de la Calle" en Quito. Universidad Politécnica Salesiana. https://dspace.ups.edu.ec/ 
Landes,D. (1998). The Wealth and poverty on Nations: Why some are so rich and some so poor, New York, W.W. Norton \& Company.

North D, (1990). Institutions, Institutional Change and Economic Development, Cambridge, Cambridge University Press.

Massey, D. (1995). Spatial Divisions of Labour: Social Structures and the Geography of Production. Macmillan International Higher Education.

Mendoza, N. (2018). Metodología de gestión estratégica en el planeamiento de desarrollo rural, el caso comunidad mi Perú, 2017-2021. Universidad Nacional del Altiplano. http://repositorio.unap.edu.pe/handle/UNAP/6960

Montesdeoca, E. (2011). Los derechos educativos de la población infantil en Cayambe. Universidad Politécnica Salesiana. https://dspace.ups.edu.ec/

Narea, F. (2011). El microcrédito productivo en Santa Elena. Universidad Politécnica Salesiana. https://dspace.ups.edu.ec/

Navarrete, Y. (2011). El programa de atención médica infantil ante la erupción del volcán Tungurahua. Universidad Politécnica Salesiana. https://dspace.ups.edu.ec/

Oliva, J. (1997). Estructuración y reestructuración de espacios y sociedades rurales: Nuevas reflexiones sobre unos procesos no esperados. Zainak, 14, 321-337.

Picci, A. (1993). The relation between central and local powers as context for endogenous development. Seminar on Strengthening Endogenous Development Patterns in European Agriculture, Chania (Greece), 20-22 Oct 1992. https://agris.fao.org/agris-search/search.do?recordID=QC2000400070

Pike, A., Rodríguez-Pose, A., \& Tomaney, J. (2007). What Kind of Local and Regional Development and for Whom? Regional Studies, 41(9), 1253-1269. https://doi.org/10.1080/00343400701543355

Quispe Fernandez, M. (2016). Visiones del desarrollo endógeno desde las comunidades locales. Revista Perspectivas, 37, 95-122.

Santana, J. (2011). Los grupos organizados de niños trabajadores en el proyecto salesiano "Chicos de la Calle" en Santo Domingo de los Tsáchilas. Universidad Politécnica Salesiana. https://dspace.ups.edu.ec/

Solano, H. (2017). DESARROLlO ENDOGENO: AGROECOLOGÍA Y AGRICULTURA CAMPESINA. Novum Scientiarum, 2(6), 10-19.

Stohr, W. B. (1992). Local initiative networks as an instrument for the development of peripheral areas. Development Issues and Strategies in the New Europe: Local, Regional and Interregional Perspectives, 203-209. 
Torres Dávila, V. H., Herrán, J., Narea, F., Montesdeoca, E., Cazares, L., Galárraga, W., Jaramillo, E., Benítez, V., Zambrano, D., De la Fuente, M., Concha, I., Castro, Y., Santana, J., \& Benavides, L. (2011). Alternativas de vida: Trece experiencias de desarrollo endógeno en Ecuador. Abya-Yala/UPS. http://dspace.ups.edu.ec/handle/123456789/6110

Torres, V. H. (2010). Surcando el bienestar el proceso de ayuda en acción en Ecuador. Universidad Politécnica Salesiana. https://dspace.ups.edu.ec/

Vázquez, A. (2007). Desarrollo endógeno. Teorías y políticas de desarrollo territorial. Investigaciones Regionales $=$ Journal of Regional Research, 11, 183-210.

Vázquez Barquero, A. (1983). Industrialization in Rural Areas. The Spanish Case. Report. OECD meeting, Senigallia.

Vázquez Barquero, A. (2000). Desarrollo económico local y descentralización: Aproximación a un marco conceptual. https://repositorio.cepal.org//handle/11362/31392

Vázquez Barquero, A. (2018). Reflexiones teóricas sobre la relacion entre desarrollo endogeno y economia social. https://doi.org/10.33776/riesise.v1i0.3581

Weber, M. (1905 [1930]). The Protestant Ethic and the Spirit of Capitalism. Londres, Reino Unido: Allen y Unwin

Woods, M., \& McDonagh, J. (2011). Rural Europe and the world: Globalization and rural development. European Countryside, 3(3), 153-163.

Zambrano, D. (2011). El ecoturismo comunitario en Manglar alto y Colonche. Universidad Politécnica Salesiana. https://dspace.ups.edu.ec/

\section{¿Ciencia}




\section{PARA CITAR EL ARTÍCULO INDEXADO.}

Quito Cortez, S. M., Flores Aguilera, D. D., \& Castillo Ortega, Y. (2021). Estrategia de desarrollo endógeno: una propuesta para comunidades ecuatorianas. Ciencia Digital, 5(1), 93 113. https://doi.org/10.33262/cienciadigital.v5i1.1518

\section{$\left\lfloor\begin{array}{l}\text { Ciencia } \\ \text { Digital } \\ \varepsilon_{\text {ddtouat }}\end{array}\right.$}

El artículo que se publica es de exclusiva responsabilidad de los autores y no necesariamente reflejan el pensamiento de la Revista Ciencia Digital.

El artículo queda en propiedad de la revista y, por tanto, su publicación parcial y/o total en otro medio tiene que ser autorizado por el director de la Revista Ciencia

Digital.
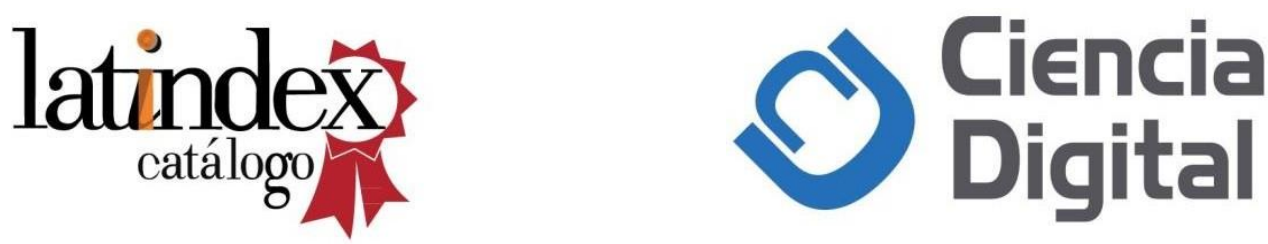